\title{
Lnc-ing RNA Expression with Disease Pathogenesis: MALAT1 and ANRIL in Ulcerative Colitis
}

\author{
Maria Serena Longhi ${ }^{1}$ Efi Kokkotou ${ }^{2}$
}

Published online: 30 March 2020

(c) Springer Science+Business Media, LLC, part of Springer Nature 2020

A wealth of studies has provided increasingly robust evidence of the involvement of long noncoding RNAs (lncRNAs) in the pathogenesis of several disease processes. lncRNAs are conventionally defined as transcripts longer than 200 nucleotides that lack protein coding potential. According to the latest version of the NONCODE database, although they can originate from $>96,000$ genes in the form of $>172,000$ transcripts, only a small percentage of lncRNAs has been annotated. They are localized primarily in the nucleus although they can also be present in the cell cytoplasm and in the extracellular fluids. IncRNAs exert their functions through interactions with chromatin, transcription factors, RNA, and proteins. In inflammatory bowel disease (IBD), although several studies reported differential expression of lncRNAs in ulcerative colitis (UC) or in Crohn's disease (CD), including DQ786343, ANRIL(CDKN2BAS1), IFNG-AS1, H19, BC012900, MALAT1, LINC01272, HNF4A-AS1, RP11-44K6.2, CCAT1, GAS5, DIO3OS, and KIF9-AS1 [1, 2], the degree of overlap among the dysregulated IncRNAs that have been identified thus far is limited, potentially owing to varying methodological approaches to lncRNA analysis, characteristics of the individual patient cohorts, and most importantly, the small number of samples analyzed.

In this issue of Digestive Diseases and Sciences, Zhu and colleagues [3] using a targeted approach examined expression of the lncRNA MALAT (metastasis-associated lung adenocarcinoma transcript1; synonyms HCN, LINC00047, MALAT-1, NCRNA00047, noncoding nuclear-enriched abundant transcript [NEAT]2, PRO1073, and mascRNA)

Efi Kokkotou

ekokkoto@bidmc.harvard.edu

1 Department of Anesthesia, Critical Care and Pain Medicine, Harvard Medical School, Boston, MA, USA

2 Department of Medicine, Division of Gastroenterology, Beth Israel Deaconess Medical Center, Harvard Medical School, Boston, MA, USA and lncRNA ANRIL (antisense noncoding RNA in the INK4 locus; synonyms CDKN2B-AS, CDKN2BAS, NCRNA00089, PCAT12, RP11-145E5.4, and p15AS) in UC using well-characterized samples from a cohort of 76 newly diagnosed and treatment-naïve UC patients and 76 gender- and age-matched healthy volunteers of the same ethnicity. The selection of MALAT1 and ANRIL was based on their previous RNA sequencing data, in which MALAT1 and ANRIL were inversely correlated. The authors reported significant upregulation of MALAT1 and ANRIL in the colonic mucosa of UC patients compared with controls, which for MALAT1 was also reflected in plasma levels. Furthermore, colonic expression of MALAT1 and ANRIL was strongly correlated among the UC patients, a relation that was not apparent among healthy controls. Though the study by Zhu et al. analyzed a relatively large and well-controlled cohort of UC patients who were newly diagnosed and treatmentnaïve, and studied both colonic and plasma lncRNA levels, it reported the upregulation of ANRIL in contrast to several previous studies involving the analysis of ileal and colonic samples from CD as well as from UC patients, where its expression was downregulated $[1,4,5]$. Nevertheless, a more recent study using Q-PCR analysis similar to the Zhu study also reported upregulation of ANRIL in the colonic mucosa of UC patients [6]. These divergent results regarding the regulation of ANRIL in UC might be attributed to several factors, including disease severity, concomitant medications, or even the gene expression normalization process. Another plausible explanation that might account at least in part for such discrepancies is the observation that there are 17 splice variants for ANRIL that have been associated with 40 disease phenotypes, including IBD [1]. Indeed, in the study by Haberman et al., the investigators mapped lncRNA expression within $500 \mathrm{~kb}$ of genomic regions flanking IBD susceptibility loci, finding that 41 of the differentially expressed IncRNAs in CD, among them ANRIL, were localized within 47 such risk variants for IBD. ANRIL was one of their top 10 downregulated genes in the ileum of 
treatment-naïve early-onset CD patients (downregulated by 7.4-fold, co-expressed with 411 other genes) [4]. The same is true for MALAT1, which has three isoforms and has been associated with five disease phenotypes, including colon cancer. It is not clear which isoforms were measured in the Zhu study.

In the study by Zhu et al., about $50 \%$ of UC patients had MALAT1 or ANRIL expression levels that were higher compared to the distribution of values in healthy controls. This finding might indicate that those patients had higher disease activity or perhaps represent a distinct subgroup of UC patients with a different disease prognosis, response to treatment, or who are at higher risk of malignancy. Ge et al [7] studied 101 patients with CD (42 with active disease and 59 in remission) and 67 healthy controls, finding that patients with active disease had lower mucosal ANRIL levels compared with healthy controls, whereas $C D$ patients in remission had intermediate levels. Furthermore, they described an inverse correlation between ANRIL expression and TNF- $\alpha$, IL-17, IL-23, and IFN- $\gamma$ expression, in contrast to a positive correlation with IL-10. Most importantly, they report that patients who subsequently responded clinically to anti-TNF treatment exhibited significant upregulation of ANRIL at week 12 post-treatment, though baseline levels were similar between anti-TNF responders and non-responders. In a different study, upregulation of IncRNA growth arrest-specific (GAS) 5 was associated with an unfavorable response to corticosteroid treatment in pediatric IBD [8]. Another group analyzed 941 cases with CD reporting that lncRNA RP11-679B19.1 isoform as a disease-modifying genetic variant associated with fibrostenotic CD [9].

From a mechanistic point of view, the positive correlation of ANRIL and MALAT1 mucosal expression levels in the Zhu study prompted further investigation of the relationship between MALAT1 and ANRIL using LPS-primed primary colonic epithelial cells. They found that overexpression of MALAT1 upregulated ANRIL, whereas the reverse was not true, suggesting for the first time that MALAT1 might act upstream of ANRIL [3]. Furthermore, MALAT1 or ANRIL overexpression almost doubled the apoptosis index in the same cells [3]. Yet, in the apoptosis experiments described in the Zhu study, cell proliferation rates were not measured in parallel. A previous study reported increased proliferation of Caco 2 cells when ANRIL was inhibited [5]. Moreover, studies in the cancer field have shown, for example, that MALAT1 overexpression promotes proliferation, migration, and invasion in cancers including colon, gastric, and pancreatic at least in part via PI3/AKT activation.

Given the upregulation of MALAT1 and ANRIL in UC shown by Zhu et al., it would be of interest to investigate, particularly in primary colonic epithelial cells, how pro-inflammatory stimuli such as LPS or IL- $1 \beta$ regulate endogenous MALAT1 or ANRIL and to what extent there was evidence of co-regulation. In a previous study, treatment of Caco 2 cells with IL-1 $\beta$ downregulated ANRIL [4]. Likewise, although treatment of HT29 colonic epithelial cells with TNF- $\alpha$ or IL- $1 \beta$ downregulated ANRIL [10], such effects might be cell type and context specific since in vascular endothelial cells treatment with $\mathrm{TNF}-\alpha$ resulted in NFKB-dependent upregulation of ANRIL [11]. Treatment with LPS of differentiated THP1 monocytes induced MALAT1 expression. In turn, MALAT1, by interacting with the NFKB subunits in the nucleus, attenuated the expression of TNF- $\alpha$ and IL-6 [11].

More mechanistic studies are needed to elucidate the contributions of MALAT1 and ANRIL to the pathogenesis of IBD, and further delineation of the cellular and subcellular localization of MALAT1 and ANRIL in the IBD context will greatly facilitate this process. As an example, Haberman et al. [4] investigated the function of the top upregulated or downregulated genes in CD resulting from RNA sequencing analysis, LINC01272 and HNF4A-AS1, respectively, by interrogating their tissue- and cell-specific expression patterns using publicly available RNA sequencing data. They found HNFA-AS1-specific expression in the small intestine, colon, and Caco 2 cells in contrast to LINC01272 expression, which was restricted to the myeloid lineage. Those findings were subsequently confirmed in paraffin-embedded tissue by in situ hybridization in patient samples. MALAT1 expression appears to be ubiquitous, whereas ANRIL expression is limited to T-cells, macrophages, colonic epithelial cells, vascular endothelial cells, macrophages, muscle, and fat. Expression of MALAT1 or ANRIL in intestinal epithelial cells might indicate involvement in epithelial barrier function; indeed, ANRIL primarily localizes to the colonic epithelium in normal colonic tissue [5]. ANRIL overexpressing experiments showed that it might affect epithelial paracellular permeability, at least in part by modulating the expression of the tight junctional protein claudin-2, a surrogate marker for paracellular permeability [5]. Besides regulating the expression of tight junction-associated proteins, IncRNAs have also been implicated in autophagy, control of Paneth and goblet cell numbers, and in mucosal repair [12]. In addition to effects on epithelial cells, it is likely that MALAT 1 or ANRIL might promote an IBD phenotype by acting directly on immune cells, as has been described for other disease entities [13]. Upon confirmation, such an immunomodulatory function, in particular if it is combined with mucosal healing properties, might reveal dysregulated lncRNAs like MALAT or ANRIL as desirable therapeutic targets in IBD.

Though IncRNAs have been studied extensively in cancer and cardiovascular disease as diagnostic or prognostic circulating biomarkers, their association with inflammatory bowel disease has just begun to emerge. Given the translational implications of the study by Zhu and of similar studies, it 
would be of importance to validate such results in a larger population of UC patients and controls in order to determine whether plasma levels of MALAT1 can contribute to disease diagnosis, monitoring or response to treatment.

\section{References}

1. Zacharopoulou E, Gazouli M, Tzouvala M, Vezakis A, Karamanolis G. The contribution of long non-coding RNAs in Inflammatory. Bowel Diseases Dig Liver Dis. 2017;49:1067-1072.

2. Yarani R, Mirza AH, Kaur S, Pociot F. The emerging role of lncRNAs in inflammatory bowel disease. Exp Mol Med. 2018;50:161.

3. Zhu M, Xie J. LncRNA MALAT1 promotes ulcerative colitis by upregulating lncRNA ANRIL. Dig Dis Sci. (Epub ahead of print). https://doi.org/10.1007/s10620-020-06093-w.

4. Haberman Y, BenShoshan M, Di Segni A, et al. Long ncRNA landscape in the ileum of treatment-naive early-onset crohn disease. Inflamm Bowel Dis. 2018;24:346-360.

5. Rankin CR, Lokhandwala ZA, Huang R, Pekow J, Pothoulakis C, Padua D. Linear and circular CDKN2B-AS1 expression is associated with inflammatory bowel disease and participates in intestinal barrier formation. Life Sci. 2019;231:116571.

6. Qiao C, Yang L, Wan J, et al. Long noncoding RNA ANRIL contributes to the development of ulcerative colitis by miR-323b-5p/ TLR4/MyD88/NF-kappaB pathway. Biochem Biophys Res Commun. 2019;508:217-224.

7. Ge Q, Dong Y, Lin G, Cao Y. Long noncoding RNA antisense noncoding RNA in the INK4 locus correlates with risk, severity, inflammation and infliximab efficacy in crohn's disease. Am J Med Sci. 2019;357:134-142.

8. Lucafo M, Di Silvestre A, Romano M, et al. Role of the long noncoding RNA growth arrest-specific 5 in glucocorticoid response in children with inflammatory bowel disease. Basic Clin Pharmacol Toxicol. 2018;122:87-93.

9. Visschedijk MC, Spekhorst LM, Cheng SC, et al. Genomic and expression analyses identify a disease-modifying variant for fibrostenotic crohn's disease. J Crohns Colitis. 2018;12:582-588.

10. Wu F, Huang Y, Dong F, Kwon JH. Ulcerative colitis-associated long noncoding RNA, BC012900, regulates intestinal epithelial cell apoptosis. Inflamm Bowel Dis. 2016;22:782-795.

11. Mao X, Su Z, Mookhtiar AK. Long non-coding RNA: a versatile regulator of the nuclear factor-kappaB signalling circuit. Immunology. 2017;150:379-388.

12. Yu TX, Chung HK, Xiao L, et al. Long noncoding RNA H19 impairs the intestinal barrier by suppressing autophagy and lowering paneth and goblet cell function. Cell Mol Gastroenterol Hepatol. 2019. https://doi.org/10.1016/j.jcmgh.2019.12.002.

13. Gupta SC, Awasthee N, Rai V, Chava S, Gunda V, Challagundla KB. Long non-coding RNAs and nuclear factor-kappaB crosstalk in cancer and other human diseases. Biochim Biophys Acta Rev Cancer. 2020;1873:188316.

Publisher's Note Springer Nature remains neutral with regard to jurisdictional claims in published maps and institutional affiliations. 